\title{
Isolation, Characterization and Transcriptome Analysis of a Cytokinin Receptor Mutant Osckt1 in Rice
}

\author{
Wona Ding ${ }^{1+}$, Huishan Tong ${ }^{2 \dagger}$, Wenjuan Zheng ${ }^{1}$, Jing Ye ${ }^{1}$, Zhichong Pan ${ }^{1}$, Botao Zhang ${ }^{\text {* }}$ \\ and Shihua Zhu' ${ }^{*}$
}

${ }^{1}$ College of Science and Technology, Ningbo University, Ningbo, China, ${ }^{2}$ School of Marine Sciences, Ningbo University, Ningbo, China, ${ }^{3}$ Cixi Institute of Biomedical Engineering, Ningbo Institute of Materials Technology and Engineering, Chinese Academy of Sciences, Ningbo, China

\section{OPEN ACCESS}

Edited by:

Keqiang Wu,

National Taiwan University, Taiwan

Reviewed by:

Stefan De Folter,

CINVESTAV, Mexico

Sudip Kundu,

University of Calcutta, India

${ }^{*}$ Correspondence:

Botao Zhang

zhangbotao@nimte.ac.cn

Shihua Zhu

zhushihua@nbu.edu.cn

${ }^{\dagger}$ These authors have contributed equally to this work.

Specialty section: This article was submitted to Plant Genetics and Genomics,

a section of the journal

Frontiers in Plant Science

Received: 30 October 2016

Accepted: 16 January 2017

Published: 31 January 2017

Citation:

Ding $W$, Tong $H$, Zheng $W$, Ye J,

Pan Z, Zhang B and Zhu S (2017)

Isolation, Characterization and Transcriptome Analysis of a

Cytokinin Receptor Mutant Osckt1

in Rice. Front. Plant Sci. 8:88.

doi: 10.3389/fpls.2017.00088
Cytokinins play important roles in regulating plant development, including shoot and root meristems, leaf longevity, and grain yield. However, the in planta functions of rice cytokinin receptors have not been genetically characterized yet. Here we isolated a rice mutant, Osckt1, with enhanced tolerance to cytokinin treatment. Further analysis showed that Osckt1 was insensitive to aromatic cytokinins but responded normally to isoprenoid and phenylurea-type cytokinins. Map-based cloning revealed that the mutation occurred in a putative cytokinin receptor gene, histidine kinase 6 (OsHK6). OsCKT1 was found to be expressed in various tissues throughout the plant and the protein was located in the endoplasmic reticulum. In addition, whole-genome gene expression profiling analysis showed that OsCKT1 was involved in cytokinin regulation of a number of biological processes, including secondary metabolism, sucrose and starch metabolism, chlorophyll synthesis, and photosynthesis. Our results demonstrate that OsCKT1 plays important roles in cytokinin perception and control of root development in rice.

Keywords: cytokinin, histidine kinase, transcriptome, rice, Oryza sativa

\section{INTRODUCTION}

Cytokinin, a class of adenine derivatives, regulates many processes in plants, such as tissue and organ development and response to environmental stimuli (Mok and Mok, 2001; Werner and Schmulling, 2009). Cytokinin signaling is transducted by a two-component system (TCS) through a His-to-Asp phosphorelay (Heyl and Schmulling, 2003; Kakimoto, 2003). TCSs were originally identified in bacteria, and in the basal form they involve a receptor kinase that autophosphorylates on a conserved His residue in response to an environmental stimulus and the phosphate is then transferred to a conserved Asp residue of a response regulator (RR), which subsequently modulates its downstream signaling in the pathway (Stock et al., 2000; Gao and Stock, 2009). In plants the TCS consists of a multistep phosphorelay, i.e., His to Asp to His to Asp (Hutchison and Kieber, 2002; Sheen, 2002; Kakimoto, 2003; Ferreira and Kieber, 2005). Cytokinin is first perceived by hybrid histidine kinase (HK) receptors, mainly localized in endoplasmic reticulum (ER), and results in autophosphorylation (Anantharaman and Aravind, 2001; Caesar et al., 2011; Lomin et al., 2011; Wulfetange et al., 2011). After an intramolecular phosphotransfer, 
the phosphorylation signal is transmitted to His-containing phosphotransfer proteins (HPs), and then they translocate to the nucleus (Punwani et al., 2010). In the nucleus, type-B RRs are activated by HPs through phosphorylation and they subsequently initiate the transcription of their target genes, including type-A RRs, a class of negative regulators of cytokinin signaling (Hwang and Sheen, 2001; Deruere and Kieber, 2002; To et al., 2004).

The system is extensively studied mainly in Arabidopsis. Three HKs, AHK2, AHK3, and AHK4, function redundantly as cytokinin receptors (Inoue et al., 2001; Ueguchi et al., 2001; Higuchi et al., 2004). The ahk2 ahk3 mutant showed fewer leaf cells, reduced chlorophyll content, a strongly enhanced root system and increased branching, and the ahk2 ahk3 ahk4 mutant showed severe shoot and root defect and very low fertility, with a reduction in meristem size and activity, seeds of the ahk2 ahk3 ahk4 mutant were more than twice as large as wild-type (WT; Higuchi et al., 2004; Riefler et al., 2006). A number of Arabidopsis RRs (ARRs) have also been characterized. Over-expression study of all type-A RRs found that they were regulated by both the cytokinin and proteasome pathways and executed distinctive functions in plant growth and development (Ren et al., 2009). ARR15 and ARR16 were shown to play distinct roles in roots (Kiba et al., 2002, 2003). Over-expression of ARR22 in Arabidopsis resulted in dwarf phenotypes and poorly developed root systems (Kiba et al., 2004). RR2 was shown to control leaf longevity through cytokinin-mediated phosphorylation (Kim et al., 2006). Three type-B RRs, ARR1, ARR10, and ARR12, were found to be key players in cytokinin regulation of root protoxylem differentiation, lateral root formation, chlorophyll levels, and cytokinin primary response (Mason et al., 2005; Yokoyama et al., 2007). arr1 arr10 arr 12 plants showed very severe growth defect which was highly analogous to the ahk2 ahk3 ahk4 triple mutant (Ishida et al., 2008).

The conserved cytokinin TCS also exist in rice (Ito and Kurata, 2006; Du et al., 2007; Schaller et al., 2007). The rice genome is predicted to have four HKs, two HPs, 13 type-B RRs and 10 type-A RRs. However, the signaling pathway is relatively less characterized in rice compared to Arabidopsis. Only a few components were genetically characterized in detail. The two HPs in rice were found to be positive regulators of the cytokinin signaling pathway and played different roles in salt and drought tolerance (Sun et al., 2014). Over-expression of OsRR6 resulted in dwarf phenotypes with poorly developed root systems and panicles (Hirose et al., 2007). Recently, OsHK3 was reported to play a role in the regulation of ABA-induced antioxidant defense and in the feedback regulation of $\mathrm{H}_{2} \mathrm{O}_{2}$ production in ABA signaling when transiently expressed in protoplasts (Wen et al., 2015). Another study also proposed that OsHK3 was similar to the osmosenser AtHK1 in Arabidopsis based on protein structure analysis (Kushwaha et al., 2014). OsHK6 was found to be a cytokinin receptor with preferential affinity for isopentenyladenine (iP; Choi et al., 2012). However, there is still no genetic characterization of rice cytokinin receptors reported yet.

Here we provided the first piece of genetic evidence to show the in planta function of a cytokinin receptor in rice using a loss-of-function mutant. We also conducted wholegenome expression profiling study to analyze its role in cytokinin regulation of rice root development.

\section{MATERIALS AND METHODS}

\section{Plant Materials and Growth Conditions}

The Osckt1 mutant was isolated from an ethyl methanesulfonate mutagenized (EMS)-mutagenized rice mutant library (Oryza sativa L. Japonica cV Xiushui63) in culture solution prepared as described with $0.2 \mu \mathrm{M}$ 6-Benzylaminopurine (BA; Yoshida et al., 1976). After being germinated in water for 2 days in the dark, phenotypic characterization of the WT and mutant was performed in a growth chamber at $30 / 22^{\circ} \mathrm{C}$ (day/night) and 60 $70 \%$ humidity with a photoperiod of $12 \mathrm{~h}$. More than 30 plants of each genotype were used for each treatment.

\section{Mapping and Cloning of OsCKT1}

A mapping population of $225 \mathrm{~F}_{2}$ plants was generated from crosses between the homozygous Osckt1 mutant and Kasalath (Oryza sativa L. Indica). $\mathrm{F}_{2}$ plants exhibiting short roots and abolished lateral roots were selected for mapping of the mutant locus of OsCKT1. OsCKT1 was mapped using SSR markers. The candidate gene between markers flanking both sides of the mutant locus was amplified by PCR from both the WT and Osckt1 plants for sanger sequencing analysis. The genomic DNA and mRNA regions of the OsCKT1 gene were amplified and sequenced using the forward and reverse primers 5'-GGGGAAGAAGGAGGAGGAGTAGATT and 5'CCACTAGCCAGACCATCATCATACC.

\section{Construction of Vectors and Plant Transformation}

The coding region of OsCKT1 mRNA was isolated by PCR amplification with the primers OsCKT1F KpnI (5'-AAA GGTACCGGGGAAGAAGGAGGAGGAGTA) and OsCKT1R SalI (5'-AAAGTCGACCCACTAGCCAGACCATCATCA). The PCR product was ligated into the pUCM-T vector (Takara) and sequenced. Then the fragment was excised from the pUCM-T vector by KpnI and SalI digestion and ligated into the corresponding site of pCAMBIA1301(35S). A 2071 bp promoter of OsCKT1 was obtained by PCR using primers: 5'-AAAGTCGACTTATTGCCCAAAATGCCCCTC (containing a SalI recognition site) and 5'-AAAGGTA CCATCCCCCCTCCCTCTCAGAAAT (containing a KpnI recognition site). The resulting DNA fragment was inserted into the vector pCAMBIA1300NH-GUS via SalI/KpnI sites to create a transcriptional fusion of the OsCKT1 promoter and the $\beta$-glucuronidase (GUS) coding sequence, OsCKT1p::GUS. The above constructs were used for Agrobacterium tumefaciensmediated rice transformation of WT or mutant materials as described (Chen et al., 2003).

\section{Histochemical GUS Staining Analysis}

Histochemical GUS analysis was performed as previously described (Ding et al., 2015). Transgenic plant samples and 
freehand cross-sections of the stem base were incubated with GUS staining solution (100 mmol $\mathrm{m}^{-1} \mathrm{NaH}_{2} \mathrm{PO}_{4}$ buffer $\mathrm{pH} 7.0$, $0.5 \%$ Triton X-100, $0.5 \mathrm{mg} \mathrm{ml}^{-1} \mathrm{X}-\mathrm{Gluc}$, and $20 \%$ methanol) overnight at $37^{\circ} \mathrm{C}$. Then, tissues were mounted on slides and photographed (Leica MZ95, Nussloch, Germany).

\section{Subcellular Localization of OsCKT1}

The full-length coding sequence of OsCKT1 with the eliminated stop codon was inserted in-frame before the coding sequence of a soluble modified green fluorescent protein (smGFP4). The OsCKT1-GFP fusion coding sequence was subcloned into the binary vector 35S-pCAMBIA1301. The resulting construct was sequenced to verify in-frame fusion and used for transient transformation of onion epidermis using a gene gun (BioRad, Hercules, CA, USA). PHF1-RFP located to the ER was co-transformed (Chen et al., 2011). The GFP and RFP were visualized using a LSM 510 laser-scanning microscope (Zeiss, Jena, Germany).

\section{RNA extraction, cDNA Library Preparation, and Digital Expression Profiling}

Total RNA was extracted from roots of 8-day old WT and Osckt1 under $0.2 \mu \mathrm{M}$ BA treatment using the RNeasy Plant Mini Kit (Qiagen, USA). Two biological replicates from each genotype were used for RNA-sequencing. RNA was quantified using the Nanodrop-2000 (ThermoFisher, USA) and RNA quality was then examined using a 2100 Bioanalyzer (Agilent Technologies, USA). High-quality RNA samples for library construction were selected based on $260 / 280 \mathrm{~nm}$ ratio and RNA integrity number (RIN) above 2.0 and 8.0, respectively. Sequencing libraries were prepared using the NEBNext Ultra RNA Library Prep Kit for Illumina (NEB, USA) according to the manufacturer's instructions. Poly-A-containing mRNA from the total RNA was isolated, purified, and fragmented. After the first and second strand cDNA synthesis and adaptor ligation, doublestranded cDNAs were then purified for end repair, dA tailing, adaptor ligation, and enrichment. Libraries were subjected to 75 cycles of single-end sequencing with the Illumina Nextseq 500 system (Illumina, USA) according to the manufacturer's instructions. The raw sequencing data have been uploaded to the SRA (Sequence Read Archive ${ }^{1}$ ) database (accession number: SRP091783).

\section{Differentially Expressed Gene Analysis}

Raw reads of fastq format were firstly processed through inhouse perl scripts. In this step, clean data were obtained by removing reads containing adapter, reads containing ploy- $\mathrm{N}$ and

\footnotetext{
${ }^{1}$ https://www.ncbi.nlm.nih.gov/sra
}
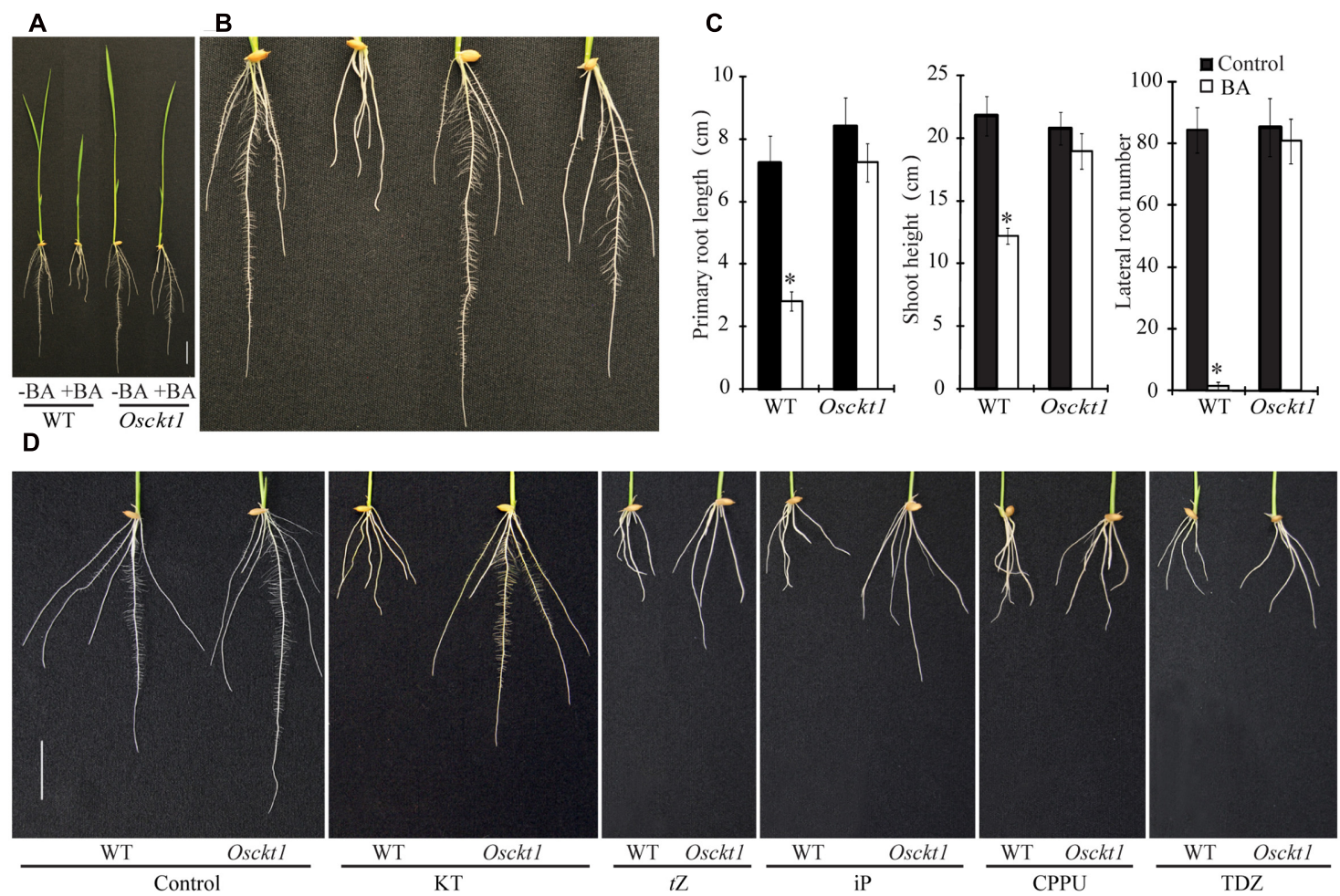

FIGURE 1 | Phenotypic characterization of the wild-type (WT) and Osckt1. (A) Growth of 8-day-old seedlings of the WT and Osckt1 without or with $0.2 \mu$ M 6-Benzylaminopurine (BA) treatment. (B) Enlarged view of roots of the WT and Osckt1 from (A). (C) Effect of BA treatment on lateral root number, shoot height, and primary root length of the WT and Osckt1. Significant differences were determined using Student's $t$-test $\left({ }^{*} P<0.05\right)$. (D) Root growth of 8 -day-old WT and Osckt1 under treatments of $0.2 \mu \mathrm{M} \mathrm{KT}, 0.05 \mu \mathrm{M} t Z, 0.1 \mu \mathrm{M}$ iP, $0.02 \mu \mathrm{M} \mathrm{CPPU}$, and $0.02 \mu \mathrm{M}$ TDZ. Bars $=2 \mathrm{~cm}$. 
low quality reads (the rate of reads which quality value $<=30$ is more than $50 \%$ ). The retained high-quality clean reads, i.e., clean reads, were then analyzed by the TopHat-Cufflinks pipeline (Trapnell et al., 2012). Briefly, cleans reads were mapped to the rice genome (MSU version $7^{2}$ ) using TopHat. Cufflinks was then used for transcriptome assembly and assessment of the FPKM value and expression difference between two genotypes. Genes differentially expressed by at least twofold between the two genotypes with a FDR adjusted $P$-value $<0.05$ were assigned as differentially expressed genes (DEGs). For gene ontology (GO) enrichment analysis, the singular enrichment analysis (SEA) tool in agriGO (Du et al., 2010) was applied with default

${ }^{2}$ http://rice.plantbiology.msu.edu/pub/data/Eukaryotic_Projects/o_sativa/ annotation_dbs/pseudomolecules/version_7.0/ parameters and a threshold FDR adjusted $P$-value $<0.05$. The function categorization of DEGs was conducted by MapMan (Thimm et al., 2004). The Kyoto Encyclopedia of Genes and Genomes (KEGG) pathway enrichment analysis was conducted by PlantGSEA (Yi et al., 2013).

\section{RESULTS}

\section{Isolation and Genetic Analysis of Osckt1}

In order to identify molecular components of the cytokinin signaling pathway in rice, a forward genetic screening was conducted using an ethylmethane sulfonate (EMS)mutagenized rice mutant library (Oryza sativa L. Japonica

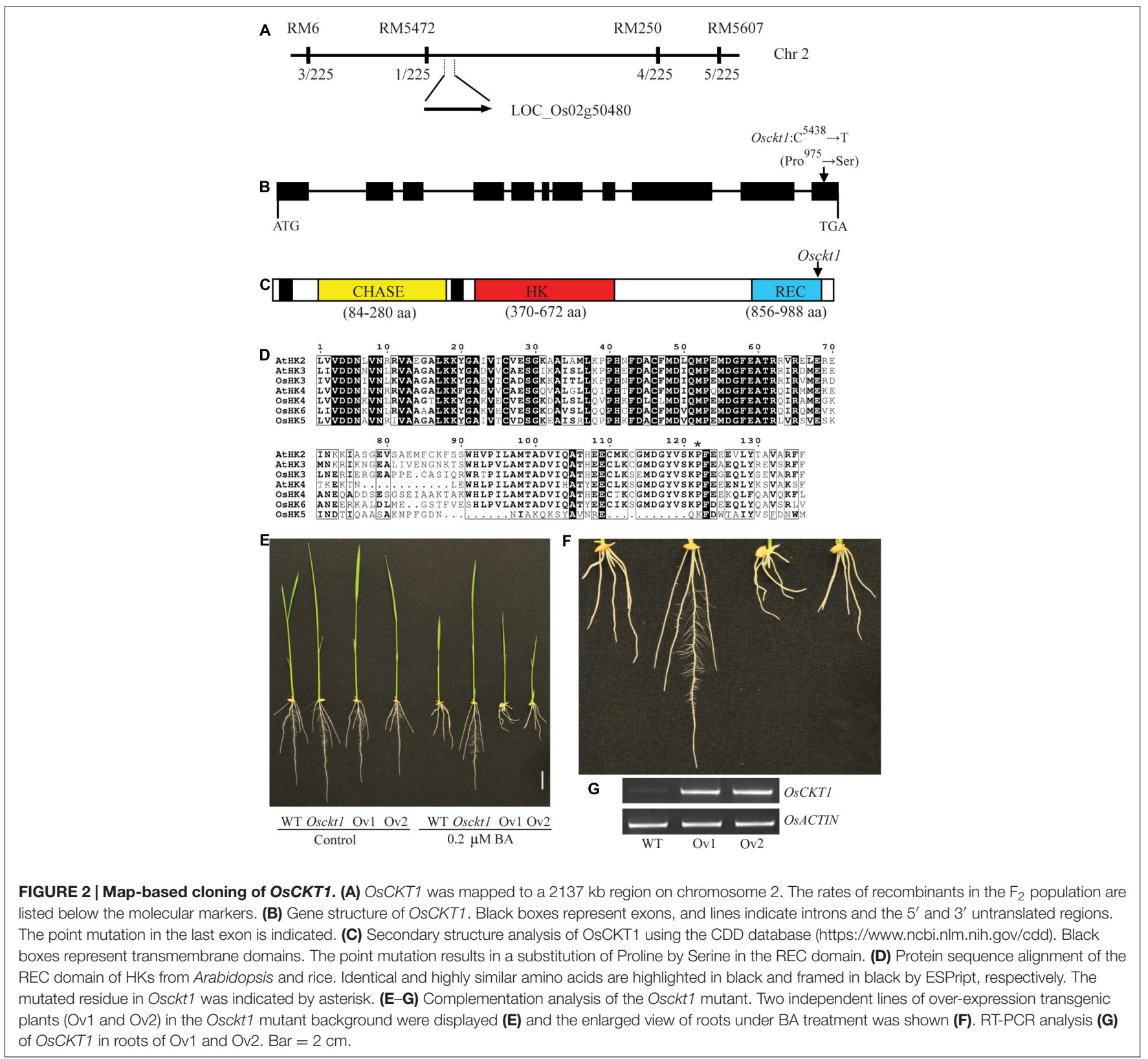


cv. Xiushui63). One mutant with decreased sensitivity to cytokinin was isolated and designated cytokinin tolerant 1 (Osckt1). Under control condition, the growth of Osckt1 was similar to the WT (Figure 1A). WT plants responded to 6-benzyladenine (BA) with significantly inhibited root and shoot growth, and especially almost no lateral root at 8 days after germination (Figures 1A-B). By contrast, such response almost disappeared in Osckt1 (Figures 1A-C). When treated with BA, root and shoot growth of Osckt1 was only slightly but not significantly reduced, and the initiation and growth of lateral roots was comparable to control condition.

The response of Osckt1 to other cytokinins was also analyzed, including kinetin (KT), trans-Zeatin ( $t \mathrm{Z})$, iP, $N$-(2-chloro-4pyridyl)- $N^{\prime}$-phenylurea (CPPU) and thidiazuron (TDZ). Among them, Osckt1 was found to be insensitive to KT only compared with the WT (Figure 1D).

\section{Cloning of OsCKT1}

A $\mathrm{F}_{2}$ population was developed by crossing the Osckt1 mutant with the WT Kasalath (Indica). The $\mathrm{F}_{1}$ seedlings showed similar sensitivity to $\mathrm{BA}$ as the WT and their $\mathrm{F}_{2}$ progenies displayed segregation of WT and Osckt1 phenotypes at a ratio of $3: 1\left(148: 52, \chi^{2}=0.35<\chi^{2}{ }_{0.05,1}=3.84\right.$, $P>0.05)$, indicating that the short root phenotype in Osckt1 is controlled by a single recessive nuclear gene. The OsCKT1 locus was first mapped to chromosome 2 between SSR markers RM5 and RM5607 using $30 \mathrm{~F}_{2}$ mutant plants (Figure 2A). Two new markers were used for fine mapping using $225 \mathrm{~F}_{2}$ mutant plants. The OsCKT1 gene was further mapped to a $2137 \mathrm{~kb}$ region between RM5472 and RM250 (Figure 2A).

The genome sequence of rice between these markers was searched for genes that could code for proteins involved in cytokinin signal transduction. Among them there was one hypothetical gene, LOC_Os02g50480, possibly encoding a cytokinin receptor, HK6. It was considered as a highly possible candidate for OsCKT1 and Sanger sequencing analysis for this gene in both the WT and Osckt1 was conducted. One single point mutation within the gene in Osckt1 was identified. The OsCKT1 gene is 5509 bp in length, and contains 11 exons and 10 introns, respectively (Figure 2B). The point mutation $\left(C^{5438}\right.$ to $\mathrm{T}$ ) occurring at the last exon of the gene resulted in an amino acid substitution (Pro ${ }^{975}$ to Ser). The protein coding region of OsCKT1 is 2994 bp and encodes a 997 amino acid protein. The protein structure is consistent with the annotation generated by
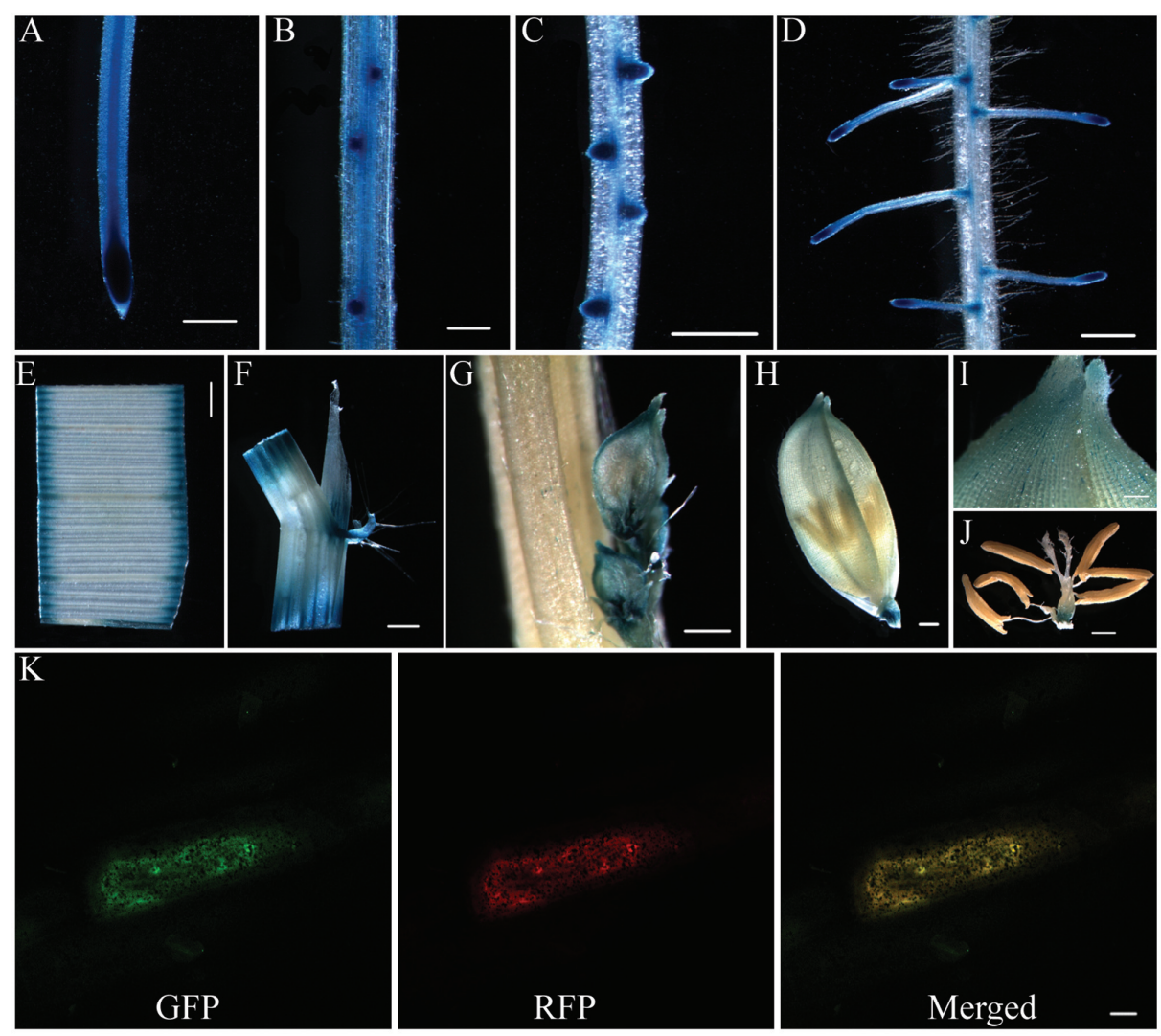

FIGURE 3 | Expression pattern of OsCKT1 and subcellular localization of OsCKT1. (A-J) Promoter- $\beta$-glucuronidase (GUS) fusion studies reveal the expression of OsCKT1 in various tissues, root tip (A), lateral root primordium (B), emerging lateral roots (C), lateral root tips (D), leaf (E), stem, ligule, and auricle (F), young spikelet (G), glume (H,I), and flower $\mathbf{( J )}$. (K) OsCKT1 targets green fluorescent protein (GFP) to ER in transiently transformed onion epidermal cells. The PHF1-RFP is used as the endoplasmic reticulum (ER) marker. (A,C-F,H,J) Bars=0.5 mm; (B,G,I) Bars=0.2 mm; (K) Bar = $20 \mu \mathrm{m}$. 
the CDD database (Conserved Domain Database ${ }^{3}$ ), with two transmembrane domains, a CHASE domain, a HK domain, and a REC domain (Figure 2C).

The CHASE domain is an extracellular cytokinin sensor and the HK domain is a dimerization and phosphoacceptor domain. Besides a phosphorylatable Asp residue for receiving the phosphoryl group from the HK domain, the REC domain is responsible for formation of homodimers or heterodimers with HPs in the process of phosphorelay and a highly conserved Pro residue within the domain was thought to be involved in formation of the hydrophobic dimerization surface (MüllerDieckmann et al., 1999; Solà et al., 1999). Protein sequence alignment analysis of the REC domain of three Arabidopsis and four rice cytokinin receptors showed that the Pro residue was highly conserved (Figure 2D). The mutation of Pro ${ }^{975}$ in Osckt1 corresponding to the conserved critical residue might explain its dramatic effect on the function of OsCKT1. It was also worth noting that the REC domain of OsHK5 only contained the first half conserved region and the other half was significantly different from others, suggesting its putatively diverged function (Figure 2D).

\section{Complementation Test of Osckt1}

Complementation analysis of the Osckt1 mutant was conducted using Agrobacterium tumefaciens-mediated transformation. The 2994 bp protein coding region of OsCKT1 was cloned into the pCAMBIA 1301 vector driven by the 35S promoter and used for transformation of Osckt1. More than twenty

${ }^{3}$ https://www.ncbi.nlm.nih.gov/cdd independent transgenic lines were obtained. The sensitivity of these transformants to BA was restored (Figures 2E-F). Insertion and expression of the transgene in two representative lines were confirmed by RT-PCR (Figure 2G).

\section{Expression Pattern and Subcellular Localization Analysis of OsCKT1}

To determine the expression pattern of OsCKT1, a $2071 \mathrm{bp}$ promoter sequence before the protein coding region of the OsCKT1 gene was fused to the GUS reporter gene. This chimeric gene cassette was use to transform WT plants via the Agrobacterium tumefaciens-mediated transformation method. Histochemical staining for GUS activity in $\mathrm{T}_{2}$ plants showed that OsCKT1 was ubiquitously expressed in plant organs, including the root, leaf, stem, ligule, auricle, young spikelet, glume, and flower (Figures 3A-J). Strong expression was observed in root tips, lateral root primordia, emerging lateral roots, and lateral root tips (Figures 3A-D).

To examine the subcellular localization of OsCKT1, a chimeric fusion gene of coding region of $\mathrm{OsCKT1}$ and the green fluorescent protein (GFP) under the control of the $35 \mathrm{~S}$ promoter was constructed and delivered into onion epidermal cells for transient expression. Fluorescence analysis showed that the fusion protein co-localized with a co-transformed ER marker (Figure 3K), indicating that OsCKT1 located in the ER.

\section{Whole-Genome Expression Analysis of Osckt1 under Cytokinin Treatment}

To further gain insight into the in planta function of Osckt1 in cytokinin signaling, digital gene expression profiling analysis
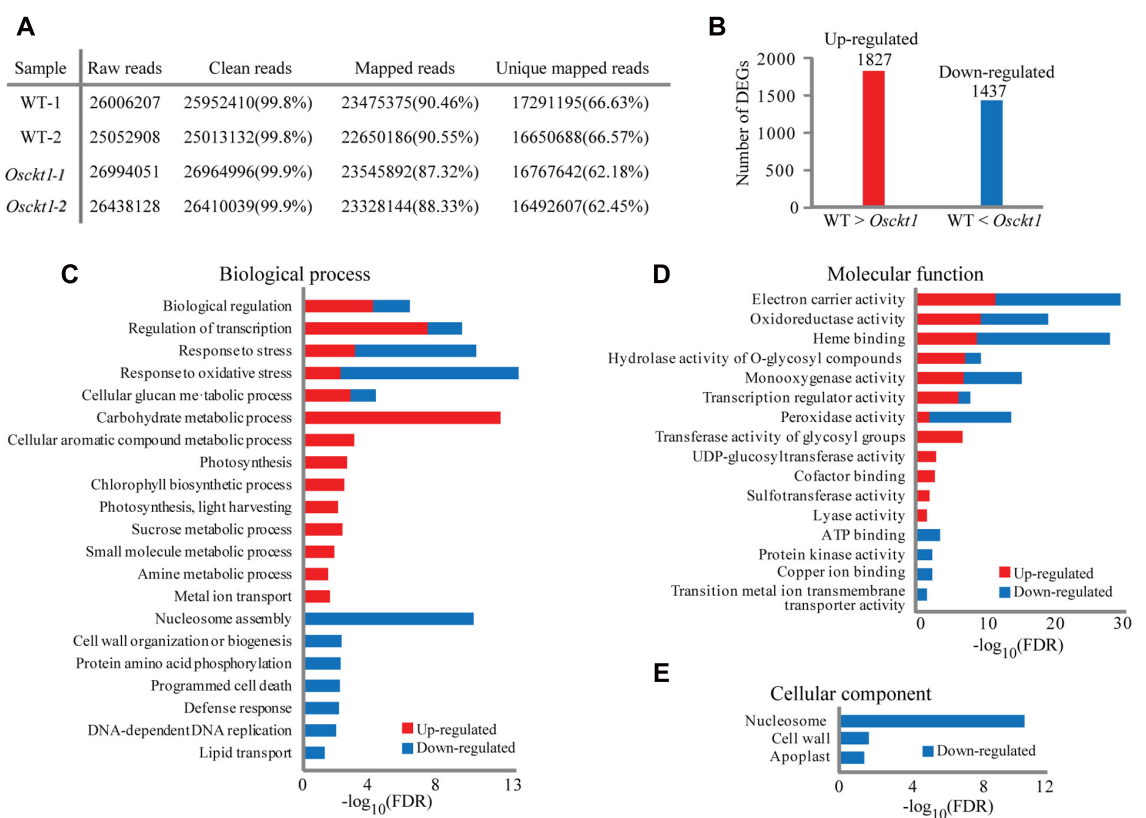

E

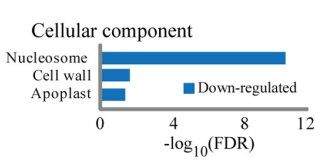

FIGURE 4 | Analysis and gene ontology (GO) enrichment of differentially expressed genes (DEGs) between the WT and Osckt1 under BA treatment by RNA-seq. (A) General information of sequencing reads and mapping. (B) The number of up- and down-regulated DEGs between the WT and Osckt1. (C-E) GO term enrichment analysis of up- and down-regulated DEGs in Biological process (C), Molecular function (D), and Cellular component (E). 
of WT and Osckt1 under BA treatment was conducted using RNA-seq. Two replicates of each genotype were used, yielding four libraries in total. Each of these libraries generated more than 25 million 75-bp single-end reads after quality control and about $62 \sim 66 \%$ of them were uniquely mapped onto the rice reference genome (Figure 4A). A total of 3264 DEGs were identified with a cut-off of $\log _{2}$ (fold change) $>=1$ and FDR adjusted $P$-value $<0.05$. Among them, $1827 \mathrm{DEG}$ showed higher expression in the WT than Osckt1 and were termed as upregulated genes, while 1437 DEGs showed lower expression in the WT than Osckt1 and were termed as down-regulated genes (Figure 4B; Supplementary Table S1).

To classify the function of DEGs, GO enrichment analysis was performed separately for up- and down-regulated DEGs using agriGO (Du et al., 2010). Within the biological process category, up-regulated DEGs were largely associated with regulation, response to stress, carbohydrate metabolism, and photosynthesis, while down-regulated DEGs were largely associated with regulation, response to stress, nucleosome assembly, cell wall, protein phosphorylation, and cell death (Figure 4C). Congruent with this, within the molecular function term both up- and down-regulated DEGs were associated with electron carrier, oxidoreductase, and heme binding (Figure 4D). Furthermore, up-regulated DEGs were specifically associated with transferase activity, while down-regulated DEGs with ATP binding and kinase activity. No up-regulated DEGs were enriched within the cellular component GO term, however, down-regulated DEGs showed association with nucleosome, cell wall, and apoplast (Figure 4E).

Kyoto Encyclopedia of Genes and Genomes pathway enrichment analysis was also conducted to identify significantly affected metabolic or signal transduction pathways among DEGs. Sixteen and four pathways were significantly enriched for up- and down-regulated DEGs, respectively (FDR $<0.05$; Figure 5A).

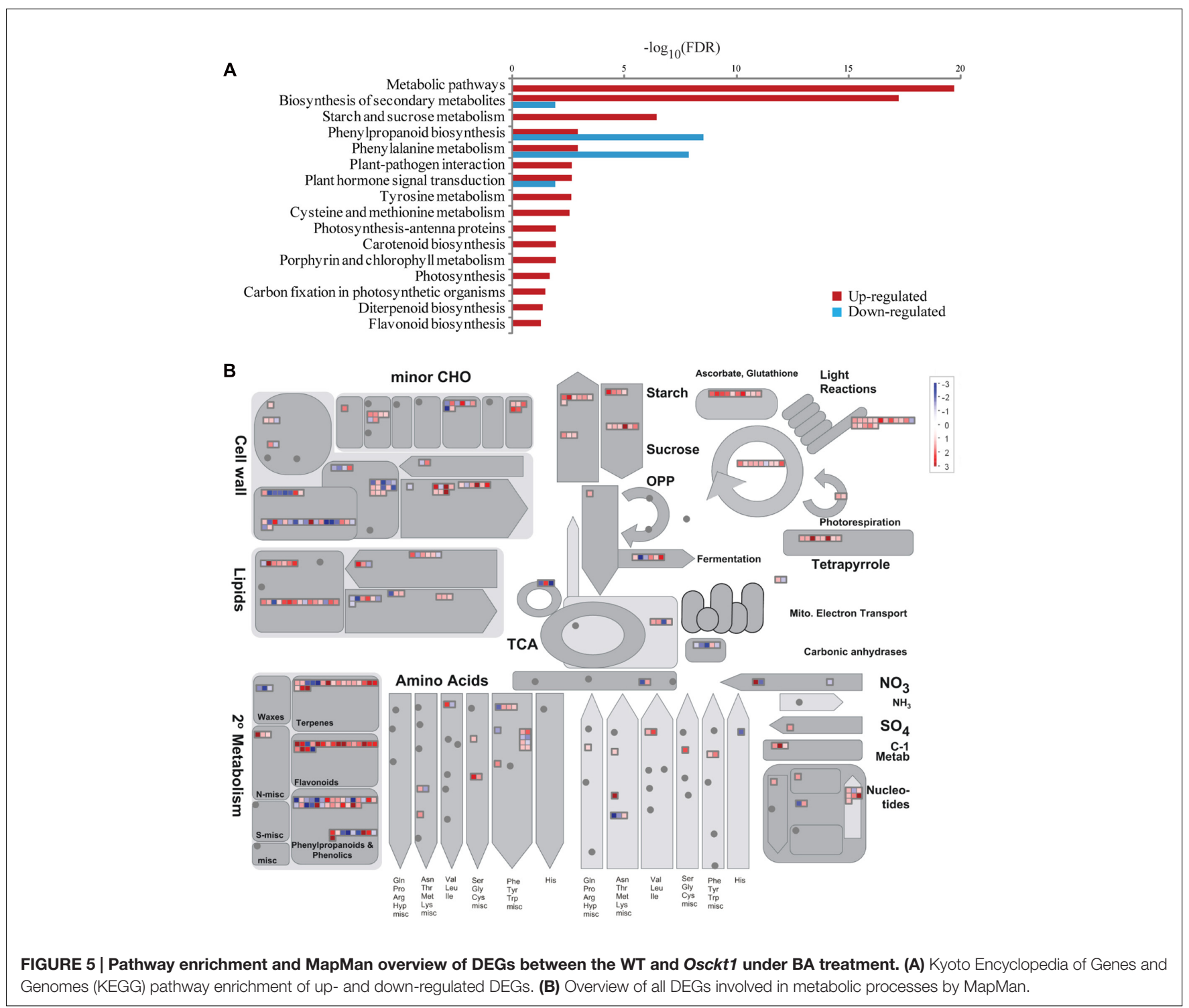


Those pathways for up-regulated DEGs mostly correlated to amino acid metabolism, secondary metabolite synthesis, hormone signaling, and photosynthesis, while down-regulated DEGs showed enrichment in phenylpropanoid biosynthesis, phenylalanine metabolism, and hormone signaling. Analysis of all DEGs using MapMan software ${ }^{4}$ further showed that DEGs involved in photosynthesis and sucrose and starch metabolism were mostly up-regulated (Figure 5B). These genes were examined in more detail. In total 22 DEGs were mapped to most steps in the calvin cycle, which is responsible for carbon fixation and sucrose and starch metabolism pathways, among which only

${ }^{4}$ http://mapman.gabipd.org one gene was down-regulated, suggesting up-regulation of these pathways under BA treatment (Figures 6A-C).

\section{DISCUSSION}

In the present study, a rice mutant Osckt 1 was isolated from a rice EMS-mutagenized population and the mutation caused loss of function of OsHK6, a cytokinin receptor. OsHK6 was proposed to be the rice ortholog of CRE1 in Arabidopsis (Du et al., 2007). Similar to its Arabidopsis counterpart, the mutant did not show any obvious growth defect under normal condition (Higuchi et al., 2004). It was previously reported that OsHK6 showed

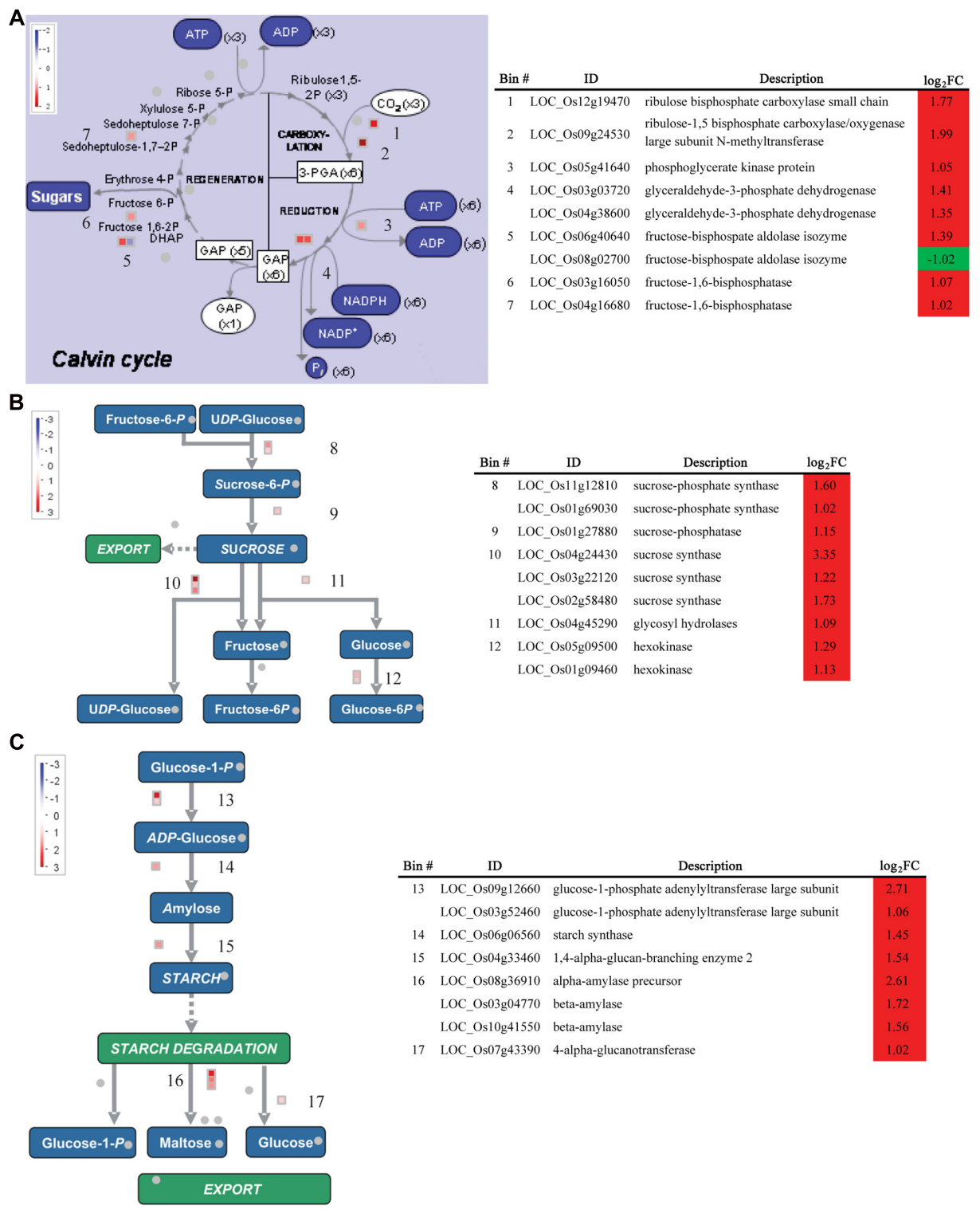

FIGURE 6 | Expression of DEGs between the WT and Osckt1 under BA treatment associated with the Calvin cycle and Starch and Sucrose metabolism. (A-C) DEGs associated with the Calvin cycle (A), sucrose (B), and starch metabolism (C) are shown, with a table showing gene names, putative functions, and fold change. The values in red and blue indicate $\log _{2}$-transformed fold increase and decrease in expression, respectively. 
TABLE 1 | Selected differentially expressed genes (DEGs) associated with hormones.

\begin{tabular}{|c|c|c|c|c|}
\hline Category & ID & Gene & Description & $\log _{2}(\mathrm{FC})$ \\
\hline \multirow[t]{8}{*}{ Biosynthesis } & LOC_Os05g47840 & IPT7 & IPP transferase & 2.14 \\
\hline & LOC_Os09g23820 & CYP72A1 & Cytochrome P450 72A1 & -2.78 \\
\hline & LOC_Os08g33300 & CYP72A1 & Cytochrome P450 72A1 & -3.35 \\
\hline & LOC_Os05g51390 & LOGL8 & Uncharacterized protein PA4923 & 1.11 \\
\hline & LOC_Os10g33900 & LOGL10 & Possible lysine decarboxylase domain containing protein & -3.76 \\
\hline & LOC_Os01g10110 & $C K X 2$ & Cytokinin dehydrogenase precursor & 5.26 \\
\hline & LOC_Os01g71310 & CKX4 & Cytokinin dehydrogenase precursor & 1.12 \\
\hline & LOC_Os01g56810 & CKX5 & Cytokinin dehydrogenase precursor & 3.13 \\
\hline \multirow[t]{10}{*}{ Conjugation } & LOC_Os04g46980 & cisZOG1 & Cis-zeatin O-glucosyltransferase & 1.72 \\
\hline & LOC_Os04g46970 & & Glucosyltransferase & 1.52 \\
\hline & LOC_Os02g28900 & & Cytokinin-O-glucosyltransferase 2 & 1.04 \\
\hline & LOC_Os04g37820 & & Cytokinin-O-glucosyltransferase 2 & 2.36 \\
\hline & LOC_Os04g25440 & & Cytokinin-O-glucosyltransferase 2 & 1.50 \\
\hline & LOC_Os02g11130 & & Cytokinin-O-glucosyltransferase 3 & 1.13 \\
\hline & LOC_Os04g44250 & & Cytokinin-O-glucosyltransferase 3 & -1.98 \\
\hline & LOC_Os07g13810 & & Cytokinin-N-glucosyltransferase 1 & 1.18 \\
\hline & LOC_Os07g13810 & & Cytokinin- $\mathrm{N}$-glucosyltransferase 1 & 1.18 \\
\hline & LOC_Os01g59100 & & Cytokinin- $N$-glucosyltransferase 1 & 1.57 \\
\hline \multirow[t]{2}{*}{ Transport } & LOC_Os09g29239 & PUP3 & Purine permease & 1.40 \\
\hline & LOC_Os01g48800 & PUP4 & Purine permease & -2.49 \\
\hline Signaling & LOC_Os02g58350 & $R R 3$ & OsRR3 type-A response regulator & -1.10 \\
\hline \multicolumn{5}{|l|}{ Auxin } \\
\hline Biosynthesis & LOC_Os01g55940 & GH3.2 & OsGH3.2 - Probable indole-3-acetic acid-amido synthetase & -1.10 \\
\hline \multirow[t]{6}{*}{ Transport } & LOC_Os11g04190 & PIN1C & Auxin efflux carrier component & -1.80 \\
\hline & LOC_Os09g38210 & PILSTb & Auxin efflux carrier component & 3.98 \\
\hline & LOC_Os09g38130 & PILS7a & Auxin efflux carrier component & 1.58 \\
\hline & LOC_Os01g50080 & MDR9 & MDR-like ABC transporter & 2.24 \\
\hline & LOC_Os03g14080 & $A \cup \times 3$ & Transmembrane amino acid transporter protein & -1.61 \\
\hline & LOC_Os10g05690 & $A \cup \times 4$ & Transmembrane amino acid transporter protein & -1.72 \\
\hline \multirow[t]{12}{*}{ Transduction } & LOC_Os01g56240 & SAUR2 & OsSAUR2 - Auxin-responsive SAUR gene family member & 1.80 \\
\hline & LOC_Os02g05050 & SAUR4 & OsSAUR4 - Auxin-responsive SAUR gene family member & 1.29 \\
\hline & LOC_Os02g07110 & SAUR6 & OsSAUR6 - Auxin-responsive SAUR gene family member & 3.10 \\
\hline & LOC_Os02g20320 & SAUR7 & OsSAUR7 - Auxin-responsive SAUR gene family member & -1.85 \\
\hline & LOC_Os04g51890 & SAUR2O & OsSAUR20 - Auxin-responsive SAUR gene family member & -1.71 \\
\hline & LOC_Os04g52670 & SAUR21 & OsSAUR21 - Auxin-responsive SAUR gene family member & 1.54 \\
\hline & LOC_Os06g48850 & SAUR27 & OsSAUR27 - Auxin-responsive SAUR gene family member & -1.45 \\
\hline & LOC_Os06g50040 & SAUR29 & OsSAUR29 - Auxin-responsive SAUR gene family member & 1.03 \\
\hline & LOC_Os09g37410 & SAUR46 & OsSAUR46 - Auxin-responsive SAUR gene family member & 1.60 \\
\hline & LOC_Os02g04810 & ARF5 & auxin response factor 5 & 1.33 \\
\hline & LOC_Os02g49160 & IAAB & OsIAA8 - Auxin-responsive Aux/IAA gene family member & 1.27 \\
\hline & LOC_Os03g58350 & IAA14 & OsIAA14 - Auxin-responsive Aux/IAA gene family member & 1.35 \\
\hline
\end{tabular}


TABLE 1 | Continued

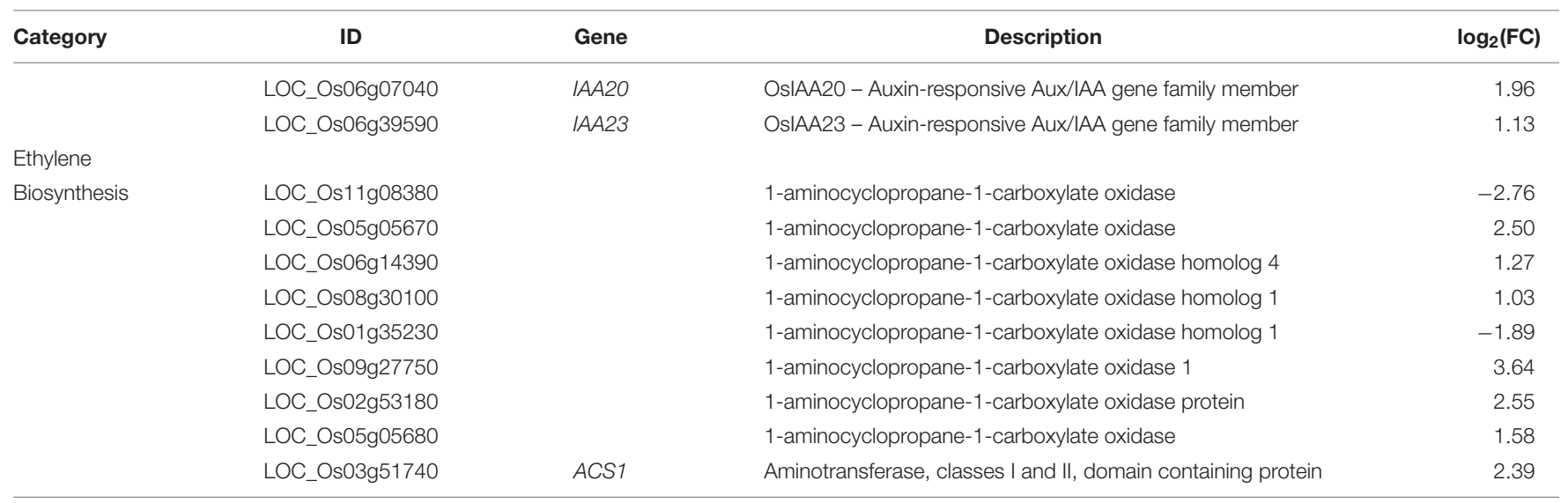

preferential affinity for iP (Choi et al., 2012). However, the in planta function of OsHK6 has not been clearly characterized. The loss-of-function mutant of OsHK6 exhibited insensitivity to BA and KT with normal root and shoot growth, especially normal lateral root initiation and elongation when treated with them, suggesting that OsHK6 was the major if not the only receptor for $\mathrm{BA}$ and $\mathrm{KT}$, two aromatic cytokinins, in rice. We further conducted rice whole-genome digital gene expression profiling analysis to compare the gene expression between WT and Osckt1 under BA treatment. GO, MapMan, and KEGG analysis showed that DEGs were significantly associated with a number of biological processes, including metabolism, hormone signal transduction, stress response, photosynthesis, etc.

Genes involved in amino acid metabolism and secondary metabolism were significantly responsive to cytokinin (Figures 5A,B). They mainly belong to two groups. One is the synthesis of phenolic secondary metabolites, including tyrosine and phenylalanine metabolism, biosynthesis of phenylpropanoid and flavonoids. It's clearly that phenylalanine metabolism and biosynthesis of phenylpropanoid both significantly enriched in the up- and down-regulated gene list by BA, indicating the complex regulation of the process. The other is biosynthesis of terpenoid, including diterpenoid and carotenoid. These results suggest that cytokinin may induce the synthesis of these compounds, which is consistent with previous reports (Deikman and Ulrich, 1995; Bhargava et al., 2013; Akagi et al., 2014).

The homeostasis of cytokinin is tightly regulated to coordinate the signaling pathways in plants (Sakakibara, 2006; Hirose et al., 2008). Adenosine phosphate isopentenyltransferase (IPT) catalyzes the fist and rate-limiting step of $\mathrm{CK}$ biosynthesis (Sakamoto et al., 2006). Then the products are hydroxylated by a cytochrome P450 monooxygenase CYP72A1 (Sakakibara, 2006). LOGs, a class of cytokinin-activating enzyme, work in the final step of bioactive cytokinin synthesis and produce freebase form of cytokinin, such as iP and tZ (Kurakawa et al., 2007). Cytokinin is then perceived by HKs, which initiate intracellular phosphotransfer to subsequent RRs (Higuchi et al., 2004). Besides, OsCKXs are cytokinin oxidase/dehydrogenases, and cytokinin glucosyltransferases catalyze the inactivation of cytokinin by $O$-glucosylation, which are responsible for removal of over-accumulated active cytokinin by degradation or conjugation, respectively (Ashikari et al., 2005; Sakakibara, 2006; Kudo et al., 2010, 2012). In our study, the expression of one IPT gene (LOC_Os03g59570), two CYP72A1 genes (LOC_Os09g23820, LOC_Os08g33300), and two LOG genes (OsLOGL3, OsLOGL10) were significantly down-regulated by BA treatment, suggesting the overall repression of cytokinin biosynthesis (Table 1). Concurrent with this, three cytokinin dehydrogenase genes (OsCKX2, OsCKX4, OsCKX5) and 13 putative cytokinin glucosyltransferase genes were significantly up-regulated by BA treatment (Table 1). Moreover, two genes (OsPUP3, OsPUP4) encoding purine permeases (PUPs)were induced and repressed, respectively. Some members of the PUP family were involved in cytokinin transport (Bürkle et al., 2003; Qi and Xiong, 2013). Several type-A RRs (OsRR4, OsRR6, OsRR9, OsRR10) were induced by BA treatment (Table 1). Type-A RRs were mainly found to be negative regulators of cytokinin signaling pathway and over-expressing $R R 6$ in rice resulted in dwarf phenotypes with poorly developed root systems (Hirose et al., 2007). The results suggest that the perception of BA in the WT may initiate the feedback signaling pathway to repress BA biosynthesis and signaling and promote BA inactivation, which help to counteract the overaccumulation of BA and maintain internal homeostasis, while in Osckt1 the inability to respond to BA treatment made these unnecessary.

Other hormones, such as auxin and ethylene, are known to closely interact with cytokinin to regulate plant development (Moubayidin et al., 2009; Schaller et al., 2015). Cytokinin suppresses auxin signaling by altering the expression of auxin efflux carrier PIN genes to control auxin transport, redistribution and downstream signaling (Staswick et al., 2005; Hwang et al., 2012; Marhavy et al., 2014). Cytokinin also induces the biosynthesis of ethylene and thus inhibits root elongation through ethylene signaling, where auxin biosynthesis, transport, signaling, and response are required (Chae et al., 2003; Stepanova et al., 2007; Ruzicka et al., 2009). In our study there are a number of auxin-related genes differentially regulated by BA treatment, including genes for auxin synthesis (YUCCA10, GH3.2, LOC_Os01g51060), transport (PIN1c, PILS7a, PILS7b, MDR9, AUX3, AUX4), and 
several SAUR gene family members and AUX/IAA genes for signal transduction (Table 1). OsGH3.2 encodes an IAA-amino synthetase which conjugates excess IAA to amino acids to suppress the action of IAA (Fu et al., 2011). Previous study in Arabidopsis showed that cytokinins repress PIN1 expression in lateral root founder cells and abolish the formation of an auxin gradient required for lateral root primordium patterning (Laplaze et al., 2007; Marhavy et al., 2011). Similarly, OsPIN1c was found to be specifically expressed in the root meristem, stele, and lateral root primordia (Wang et al., 2009). OsIAA23 was previously reported to be critical for root development, and its constitutive activation resulted in the absence of lateral and crown root primordia ( $\mathrm{Ni}$ et al., 2011). The repression of OsPINc and the induction of OsIAA23 in the WT by BA treatment might play key roles in the observed defects of root development. Moreover, one ACC synthase and six ACC oxidase genes involved in ethylene synthesis were upregulated in the WT by BA treatment (Table 1), which might result in the activation of ethylene signaling pathways and subsequently lead to inhibition of root elongation through auxin.

Chlorophyll biosynthesis and chloroplast biogenesis are positively regulated by cytokinin signaling and negatively regulated by auxin signaling in Arabidopsis roots (Kobayashi et al., 2012). Treatment of rice leaves by BA induce the expression of genes in the chlorophyll cycle and PSII-related genes, resulting in delay of senescence and the stability of photosynthetic pigment complexes (Talla et al., 2016). Consistent with this, GO enrichment and KEGG enrichment analysis in our study both showed the over-representation of genes involved in photosynthesis, chlorophyll synthesis in the up-regulated gene list (Figures $4 \mathrm{C}$ and $\mathbf{5 A}$ ).

Furthermore, a number of genes involved in the Calvin cycle and starch and sucrose metabolism were up-regulated (Figures 5B and 6A-C). For the Calvin cycle, those up-regulated genes encode rubisco-related components, phosphoglycerate kinase, glyceraldehyde-3-phosphate dehydrogenase, fructosebisphosphate aldolase, fructose-1,6-bisphosphatase, and sedoheptulose-bisphosphatase, respectively. Two genes encoding the sucrose-phosphate synthase, the key enzyme in sucrose synthesis, and one gene encoding sucrose phosphatase were highly induced by BA treatment. In addition, gene encoding sucrose synthase, glycosyl hydrolases, and hexokinase showed significant increase in expression under BA treatment. Hexokinases are sugar sensors and lead to glucoses entering the glycolytic pathway (Jang et al., 1997). Besides, genes encoding enzymes involved in both starch synthesis and degradation,

\section{REFERENCES}

Akagi, A., Fukushima, S., Okada, K., Jiang, C. J., Yoshida, R., Nakayama, A., et al. (2014). WRKY45-dependent priming of diterpenoid phytoalexin biosynthesis in rice and the role of cytokinin in triggering the reaction. Plant Mol. Biol. 86, 171-183. doi: 10.1007/s11103-0140221-x

Anantharaman, V., and Aravind, L. (2001). The CHASE domain: a predicted ligand-binding module in plant cytokinin receptors and other eukaryotic and namely ADP glucose pyrophosphorylase, starch synthase, starchbranching enzyme and amylase, were significantly induced by BA treatment. Overall, these data suggest an up-regulation of sucrose and starch biosynthesis and degradation by BA treatment.

In brief, we characterized the in planta function of a cytokinin receptor, OsHK6, using a loss-of-function mutant. Our results showed that the mutation caused tolerance of root development to BA and kinetin, suggesting that they are specifically perceived by OsHK6 in vivo. We further conducted rice whole-genome digital gene expression profiling to elucidate the underlying molecular mechanism and identified enriched functional groups involved in chlorophyll synthesis and carbon fixation, starch and sucrose metabolism, secondary metabolite synthesis, hormone signal transduction, etc. These results may improve our understanding of the functions of cytokinin signaling pathways in rice root development.

\section{AUTHOR CONTRIBUTIONS}

WD, BZ, and SZ conceived and designed the study. WD, HT, WZ, JY, ZP, and BZ performed the experiments. WD and BZ analyzed data and wrote the manuscript. All authors read and approved the manuscript.

\section{FUNDING}

This work was supported by the National Natural Science Foundation of China [grant numbers 31300246, 31371595], the Zhejiang Provincial Natural Science Foundation of China [LY17C020002, LQ16C020001], and K. C. Wong Magna Fund at Ningbo University.

\section{ACKNOWLEDGMENT}

We are grateful to Prof. Ping Wu (College of Life Science, Zhejiang University, Hangzhou, China) for providing the mutant.

\section{SUPPLEMENTARY MATERIAL}

The Supplementary Material for this article can be found online at: http://journal.frontiersin.org/article/10.3389/fpls.2017.00088/ full\#supplementary-material

bacterial receptors. Trends Biochem. Sci. 26, 579-582. doi: 10.1016/S09680004(01)01968-5

Ashikari, M., Sakakibara, H., Lin, S., Yamamoto, T., Takashi, T., Nishimura, A., et al. (2005). Cytokinin oxidase regulates rice grain production. Science 309, 741-745. doi: 10.1126/science.1113373

Bhargava, A., Clabaugh, I., To, J. P., Maxwell, B. B., Chiang, Y. H., Schaller, G. E., et al. (2013). Identification of cytokinin-responsive genes using microarray meta-analysis and RNA-Seq in Arabidopsis. Plant Physiol. 162, 272-294. doi: $10.1104 /$ pp.113.217026 
Bürkle, L., Cedzich, A., Döpke, C., Stransky, H., Okumoto, S., Gillissen, B., et al. (2003). Transport of cytokinins mediated by purine transporters of the PUP family expressed in phloem, hydathodes, and pollen of Arabidopsis. Plant J. 34, 13-26. doi: 10.1046/j.1365-313X.2003.01700.x

Caesar, K., Thamm, A. M., Witthoft, J., Elgass, K., Huppenberger, P., Grefen, C., et al. (2011). Evidence for the localization of the Arabidopsis cytokinin receptors AHK3 and AHK4 in the endoplasmic reticulum. J. Exp. Bot. 62, 5571-5580. doi: $10.1093 /$ jxb/err238

Chae, H. S., Faure, F., and Kieber, J. J. (2003). The eto1, eto2, and eto3 mutations and cytokinin treatment increase ethylene biosynthesis in Arabidopsis by increasing the stability of ACS protein. Plant Cell 15, 545-559. doi: 10.1105/ tpc.006882

Chen, J., Liu, Y., Ni, J., Wang, Y., Bai, Y., Shi, J., et al. (2011). OsPHF1 regulates the plasma membrane localization of low- and high-affinity inorganic phosphate transporters and determines inorganic phosphate uptake and translocation in rice. Plant Physiol. 157, 269-278. doi: 10.1104/pp.111.181669

Chen, S., Jin, W., Wang, M., Zhang, F., Zhou, J., Jia, Q., et al. (2003). Distribution and characterization of over 1000 T-DNA tags in rice genome. Plant J. 36, 105-113. doi: 10.1046/j.1365-313X.2003.01860.x

Choi, J., Lee, J., Kim, K., Cho, M., Ryu, H., An, G., et al. (2012). Functional identification of OsHk6 as a homotypic cytokinin receptor in rice with preferential affinity for iP. Plant Cell Physiol. 53, 1334-1343. doi: 10.1093/pcp/ pcs079

Deikman, J., and Ulrich, M. (1995). A novel cytokinin-resistant mutant of Arabidopsis with abbreviated shoot development. Planta 195, 440-449. doi: 10.1007/BF00202603

Deruere, J., and Kieber, J. J. (2002). Molecular mechanisms of cytokinin signaling. J. Plant Growth Regul. 21, 32-39. doi: 10.1007/s003440010045

Ding, W., Lin, L., Zhang, B., Xiang, X., Wu, J., Pan, Z., et al. (2015). OsKASI, a beta-ketoacyl-[acyl carrier protein] synthase I, is involved in root development in rice (Oryza sativa L.). Planta 242, 203-213. doi: 10.1007/s00425-015-2296-2

Du, L., Jiao, F., Chu, J., Jin, G., Chen, M., and Wu, P. (2007). The two-component signal system in rice (Oryza sativa L.): a genome-wide study of cytokinin signal perception and transduction. Genomics 89, 697-707. doi: 10.1016/j.ygeno.2007. 02.001

Du, Z., Zhou, X., Ling, Y., Zhang, Z., and Su, Z. (2010). agriGO: a GO analysis toolkit for the agricultural community. Nucleic Acids Res. 38, W64-W70. doi: 10.1093/nar/gkq310

Ferreira, F. J., and Kieber, J. J. (2005). Cytokinin signaling. Curr. Opin. Plant Biol. 8, 518-525. doi: 10.1016/j.pbi.2005.07.013

Fu, J., Liu, H., Li, Y., Yu, H., Li, X., Xiao, J., et al. (2011). Manipulating broad-spectrum disease resistance by suppressing pathogen-induced auxin accumulation in rice. Plant Physiol. 155, 589-602. doi: 10.1104/pp.110.163774

Gao, R., and Stock, A. M. (2009). Biological insights from structures of twocomponent proteins. Annu. Rev. Microbiol. 63, 133-154. doi: 10.1146/annurev. micro.091208.073214

Heyl, A., and Schmulling, T. (2003). Cytokinin signal perception and transduction. Curr. Opin. Plant Biol. 6, 480-488. doi: 10.1016/S1369-5266(03)00087-6

Higuchi, M., Pischke, M. S., Mähönen, A. P., Miyawaki, K., Hashimoto, Y., Seki, M., et al. (2004). In planta functions of the Arabidopsis cytokinin receptor family. Proc. Natl. Acad. Sci. U.S.A. 101, 8821-8826. doi: 10.1073/pnas.0402887101

Hirose, N., Makita, N., Kojima, M., Kamada-Nobusada, T., and Sakakibara, H. (2007). Overexpression of a type-A response regulator alters rice morphology and cytokinin metabolism. Plant Cell Physiol. 48, 523-539. doi: 10.1093/pcp/ pcm 022

Hirose, N., Takei, K., Kuroha, T., Kamada-Nobusada, T., Hayashi, H., and Sakakibara, H. (2008). Regulation of cytokinin biosynthesis, compartmentalization and translocation. J. Exp. Bot. 59, 75-83. doi: $10.1093 /$ jxb/erm 157

Hutchison, C. E., and Kieber, J. J. (2002). Cytokinin signaling in Arabidopsis. Plant Cell 14(Suppl.), S47-S59.

Hwang, I., and Sheen, J. (2001). Two-component circuitry in Arabidopsis cytokinin signal transduction. Nature 413, 383-389. doi: 10.1038/35093090

Hwang, I., Sheen, J., and Müller, B. (2012). Cytokinin signaling networks. Annu. Rev. Plant Biol. 63, 353-380. doi: 10.1146/annurev-arplant-042811-105503

Inoue, T., Higuchi, M., Hashimoto, Y., Seki, M., Kobayashi, M., Kato, T., et al. (2001). Identification of CRE1 as a cytokinin receptor from Arabidopsis. Nature 409, 1060-1063. doi: 10.1038/35059117
Ishida, K., Yamashino, T., Yokoyama, A., and Mizuno, T. (2008). Three type-B response regulators, ARR1, ARR10 and ARR12, play essential but redundant roles in cytokinin signal transduction throughout the life cycle of Arabidopsis thaliana. Plant Cell Physiol. 49, 47-57.

Ito, Y., and Kurata, N. (2006). Identification and characterization of cytokininsignalling gene families in rice. Gene 382, 57-65. doi: 10.1016/j.gene.2006.06. 020

Jang, J. C., Leon, P., Zhou, L., and Sheen, J. (1997). Hexokinase as a sugar sensor in higher plants. Plant Cell 9, 5-19.

Kakimoto, T. (2003). Perception and signal transduction of cytokinins. Annu. Rev. Plant Biol. 54, 605-627. doi: 10.1146/annurev.arplant.54.031902.134802

Kiba, T., Aoki, K., Sakakibara, H., and Mizuno, T. (2004). Arabidopsis response regulator, ARR22, ectopic expression of which results in phenotypes similar to the wol cytokinin-receptor mutant. Plant Cell Physiol. 45, 1063-1077.

Kiba, T., Yamada, H., and Mizuno, T. (2002). Characterization of the ARR15 and ARR16 response regulators with special reference to the cytokinin signaling pathway mediated by the AHK4 histidine kinase in roots of Arabidopsis thaliana. Plant Cell Physiol. 43, 1059-1066. doi: 10.1093/pcp/pcf121

Kiba, T., Yamada, H., Sato, S., Kato, T., Tabata, S., Yamashino, T., et al. (2003). The type-A response regulator, ARR15, acts as a negative regulator in the cytokininmediated signal transduction in Arabidopsis thaliana. Plant Cell Physiol. 44, $868-874$.

Kim, H. J., Ryu, H., Hong, S. H., Woo, H. R., Lim, P. O., Lee, I. C., et al. (2006). Cytokinin-mediated control of leaf longevity by AHK3 through phosphorylation of ARR2 in Arabidopsis. Proc. Natl. Acad. Sci. U.S.A. 103, 814-819. doi: 10.1073/pnas.0505150103

Kobayashi, K., Baba, S., Obayashi, T., Sato, M., Toyooka, K., Keranen, M., et al. (2012). Regulation of root greening by light and auxin/cytokinin signaling in Arabidopsis. Plant Cell 24, 1081-1095. doi: 10.1105/tpc.111.092254

Kudo, T., Kiba, T., and Sakakibara, H. (2010). Metabolism and long-distance translocation of cytokinins. J. Integr. Plant Biol. 52, 53-60. doi: 10.1111/j.17447909.2010.00898.x

Kudo, T., Makita, N., Kojima, M., Tokunaga, H., and Sakakibara, H. (2012). Cytokinin activity of cis-zeatin and phenotypic alterations induced by overexpression of putative cis-zeatin-o-glucosyltransferase in rice. Plant Physiol. 160, 319-331. doi: 10.1104/pp.112.196733

Kurakawa, T., Ueda, N., Maekawa, M., Kobayashi, K., Kojima, M., Nagato, Y., et al. (2007). Direct control of shoot meristem activity by a cytokinin-activating enzyme. Nature 445, 652-655. doi: 10.1038/nature05504

Kushwaha, H. R., Singla-Pareek, S. L., and Pareek, A. (2014). Putative osmosensor-OsHK3b-a histidine kinase protein from rice shows high structural conservation with its ortholog AtHK1 from Arabidopsis. J. Biomol. Struct. Dyn. 32, 1318-1332. doi: 10.1080/07391102.2013.818576

Laplaze, L., Benkova, E., Casimiro, I., Maes, L., Vanneste, S., Swarup, R., et al. (2007). Cytokinins act directly on lateral root founder cells to inhibit root initiation. Plant Cell 19, 3889-3900. doi: 10.1105/tpc.107.055863

Lomin, S. N., Yonekura-Sakakibara, K., Romanov, G. A., and Sakakibara, H. (2011). Ligand-binding properties and subcellular localization of maize cytokinin receptors. J. Exp. Bot. 62, 5149-5159. doi: 10.1093/jxb/err220

Marhavy, P., Bielach, A., Abas, L., Abuzeineh, A., Duclercq, J., Tanaka, H., et al. (2011). Cytokinin modulates endocytic trafficking of PIN1 auxin efflux carrier to control plant organogenesis. Dev. Cell 21, 796-804. doi: 10.1016/j.devcel. 2011.08.014

Marhavy, P., Duclercq, J., Weller, B., Feraru, E., Bielach, A., Offringa, R., et al. (2014). Cytokinin controls polarity of PIN1-dependent auxin transport during lateral root organogenesis. Curr. Biol. 24, 1031-1037. doi: 10.1016/j.cub.2014. 04.002

Mason, M. G., Mathews, D. E., Argyros, D. A., Maxwell, B. B., Kieber, J. J., Alonso, J. M., et al. (2005). Multiple type-B response regulators mediate cytokinin signal transduction in Arabidopsis. Plant Cell 17, 3007-3018. doi: 10.1105/tpc.105. 035451

Mok, D. W., and Mok, M. C. (2001). Cytokinin metabolism and action. Annu. Rev. Plant Physiol. Plant Mol. Biol. 52, 89-118. doi: 10.1146/annurev.arplant.52.1.89

Moubayidin, L., Di Mambro, R., and Sabatini, S. (2009). Cytokinin-auxin crosstalk. Trends Plant Sci. 14, 557-562. doi: 10.1016/j.tplants.2009.06.010

Müller-Dieckmann, H.-J., Grantz, A. A., and Kim, S.-H. (1999). The structure of the signal receiver domain of the Arabidopsis thaliana ethylene receptor ETR1. Structure 7, 1547-1556. doi: 10.1016/S0969-2126(00)88345-8 
Ni, J., Wang, G., Zhu, Z., Zhang, H., Wu, Y., and Wu, P. (2011). OsIAA23-mediated auxin signaling defines postembryonic maintenance of QC in rice. Plant J. 68, 433-442. doi: 10.1111/j.1365-313X.2011.04698.x

Punwani, J. A., Hutchison, C. E., Schaller, G. E., and Kieber, J. J. (2010). The subcellular distribution of the Arabidopsis histidine phosphotransfer proteins is independent of cytokinin signaling. Plant J. 62, 473-482. doi: 10.1111/j.1365313X.2010.04165.X

Qi, Z., and Xiong, L. (2013). Characterization of a purine permease family gene OsPUP7 involved in growth and development control in rice. J. Integr. Plant Biol. 55, 1119-1135. doi: 10.1111/jipb.12101

Ren, B., Liang, Y., Deng, Y., Chen, Q., Zhang, J., Yang, X., et al. (2009). Genomewide comparative analysis of type-A Arabidopsis response regulator genes by overexpression studies reveals their diverse roles and regulatory mechanisms in cytokinin signaling. Cell Res. 19, 1178-1190. doi: 10.1038/cr.2009.88

Riefler, M., Novak, O., Strnad, M., and Schmulling, T. (2006). Arabidopsis cytokinin receptor mutants reveal functions in shoot growth, leaf senescence, seed size, germination, root development, and cytokinin metabolism. Plant Cell 18, 40-54. doi: 10.1105/tpc.105.037796

Ruzicka, K., Simaskova, M., Duclercq, J., Petrasek, J., Zazimalova, E., Simon, S., et al. (2009). Cytokinin regulates root meristem activity via modulation of the polar auxin transport. Proc. Natl. Acad. Sci. U.S.A. 106, 4284-4289. doi: $10.1073 /$ pnas.0900060106

Sakakibara, H. (2006). Cytokinins: activity, biosynthesis, and translocation. Annu. Rev. Plant Biol. 57, 431-449. doi: 10.1146/annurev.arplant.57.032905.105231

Sakamoto, T., Sakakibara, H., Kojima, M., Yamamoto, Y., Nagasaki, H., Inukai, Y., et al. (2006). Ectopic expression of KNOTTED1-like homeobox protein induces expression of cytokinin biosynthesis genes in rice. Plant Physiol. 142, 54-62. doi: 10.1104/pp.106.085811

Schaller, G. E., Bishopp, A., and Kieber, J. J. (2015). The yin-yang of hormones: cytokinin and auxin interactions in plant development. Plant Cell 27, 44-63. doi: $10.1105 /$ tpc.114.133595

Schaller, G. E., Doi, K., Hwang, I., Kieber, J. J., Khurana, J. P., Kurata, N., et al. (2007). Nomenclature for two-component signaling elements of rice. Plant Physiol. 143, 555-557. doi: 10.1104/pp.106.093666

Sheen, J. (2002). Phosphorelay and transcription control in cytokinin signal transduction. Science 296, 1650-1652. doi: 10.1126/science.1071883

Solà, M., Gomis-Rüth, F. X., Serrano, L., González, A., and Coll, M. (1999). Three-dimensional crystal structure of the transcription factor PhoB receiver domain1. J. Mol. Biol. 285, 675-687. doi: 10.1006/jmbi.1998.2326

Staswick, P. E., Serban, B., Rowe, M., Tiryaki, I., Maldonado, M. T., Maldonado, M. C., et al. (2005). Characterization of an Arabidopsis enzyme family that conjugates amino acids to indole-3-acetic acid. Plant Cell 17, 616-627. doi: 10.1105/tpc. 104.026690

Stepanova, A. N., Yun, J., Likhacheva, A. V., and Alonso, J. M. (2007). Multilevel interactions between ethylene and auxin in Arabidopsis roots. Plant Cell 19, 2169-2185. doi: 10.1105/tpc.107.052068

Stock, A. M., Robinson, V. L., and Goudreau, P. N. (2000). Two-component signal transduction. Annu. Rev. Biochem. 69, 183-215. doi: 10.1146/annurev.biochem. 69.1 .183

Sun, L., Zhang, Q., Wu, J., Zhang, L., Jiao, X., Zhang, S., et al. (2014). Two rice authentic histidine phosphotransfer proteins, OsAHP1 and OsAHP2, mediate cytokinin signaling and stress responses in rice. Plant Physiol. 165, 335-345. doi: $10.1104 /$ pp.113.232629
Talla, S. K., Panigrahy, M., Kappara, S., Nirosha, P., Neelamraju, S., and Ramanan, R. (2016). Cytokinin delays dark-induced senescence in rice by maintaining the chlorophyll cycle and photosynthetic complexes. J. Exp. Bot. 67, 1839-1851. doi: 10.1093/jxb/erv575

Thimm, O., Blasing, O., Gibon, Y., Nagel, A., Meyer, S., Kruger, P., et al. (2004). MAPMAN: a user-driven tool to display genomics data sets onto diagrams of metabolic pathways and other biological processes. Plant J. 37, 914-939. doi: 10.1111/j.1365-313X.2004.02016.x

To, J. P., Haberer, G., Ferreira, F. J., Deruere, J., Mason, M. G., Schaller, G. E., et al. (2004). Type-A Arabidopsis response regulators are partially redundant negative regulators of cytokinin signaling. Plant Cell 16, 658-671. doi: 10.1105/ tpc. 018978

Trapnell, C., Roberts, A., Goff, L., Pertea, G., Kim, D., Kelley, D. R., et al. (2012). Differential gene and transcript expression analysis of RNA-seq experiments with TopHat and Cufflinks. Nat. Protoc. 7, 562-578. doi: 10.1038/nprot. 2012.016

Ueguchi, C., Sato, S., Kato, T., and Tabata, S. (2001). The AHK4 gene involved in the cytokinin-signaling pathway as a direct receptor molecule in Arabidopsis thaliana. Plant Cell Physiol. 42, 751-755. doi: 10.1093/pcp/pce094

Wang, J.-R., Hu, H., Wang, G.-H., Li, J., Chen, J.-Y., and Wu, P. (2009). Expression of PIN genes in rice (Oryza sativa L.): tissue specificity and regulation by hormones. Mol. Plant 2, 823-831. doi: 10.1093/mp/ssp023

Wen, F., Qin, T., Wang, Y., Dong, W., Zhang, A., Tan, M., et al. (2015). OsHK3 is a crucial regulator of abscisic acid signaling involved in antioxidant defense in rice. J. Integr. Plant Biol. 57, 213-228. doi: 10.1111/jipb.12222

Werner, T., and Schmulling, T. (2009). Cytokinin action in plant development. Curr. Opin. Plant Biol. 12, 527-538. doi: 10.1016/j.pbi.2009.07.002

Wulfetange, K., Lomin, S. N., Romanov, G. A., Stolz, A., Heyl, A., and Schmülling, T. (2011). The cytokinin receptors of Arabidopsis are located mainly to the endoplasmic reticulum. Plant Physiol. 156, 1808-1818. doi: 10. 1104/pp.111.180539

Yi, X., Du, Z., and Su, Z. (2013). PlantGSEA: a gene set enrichment analysis toolkit for plant community. Nucleic Acids Res. 41, W98-W103. doi: 10.1093/nar/ gkt281

Yokoyama, A., Yamashino, T., Amano, Y., Tajima, Y., Imamura, A., Sakakibara, H., et al. (2007). Type-B ARR transcription factors, ARR10 and ARR12, are implicated in cytokinin-mediated regulation of protoxylem differentiation in roots of Arabidopsis thaliana. Plant Cell Physiol. 48, 84-96.

Yoshida, S., Forno, D. A., Cock, J. H., and Gomez, K. A. (1976). "Routine procedure for growing rice plants in culture solution," in Laboratory Manual for Physiological Studies of Rice, eds S. Yoshida, D. A. Forno, J. H. Cook, and K. A. Gomez (Los Banos, CA: International Rice Research Institute), 61-66.

Conflict of Interest Statement: The authors declare that the research was conducted in the absence of any commercial or financial relationships that could be construed as a potential conflict of interest.

Copyright $(2017$ Ding, Tong, Zheng, Ye, Pan, Zhang and Zhu. This is an open-access article distributed under the terms of the Creative Commons Attribution License (CC BY). The use, distribution or reproduction in other forums is permitted, provided the original author(s) or licensor are credited and that the original publication in this journal is cited, in accordance with accepted academic practice. No use, distribution or reproduction is permitted which does not comply with these terms. 